\title{
VOID HIERARCHY AND COSMIC STRUCTURE
}

\author{
Rien van de Weygaert ${ }^{1} \&$ Ravi Sheth ${ }^{2,3}$ \\ ${ }^{1}$ Kapteyn Institute, Univ. Groningen, P.O. Box 800, 9700 AV Groningen, The Netherlands \\ 2 Dept. of Physics and Astronomy, University of Pittsburgh, 3941 O'Hara St., PA 15260, U.S.A. \\ ${ }^{3}$ NASA/Fermilab Astrophysics Group, Batavia, IL 60510-0500, U.S.A. \\ rks12@pitt.edu, weygaert@astro.rug.nl
}

\begin{abstract}
Within the context of hierarchical scenarios of gravitational structure formation we describe how an evolving hierarchy of voids evolves on the basis of two processes, the void-in-void process and the void-in-cloud process. The related analytical formulation in terms of a two-barrier excursion problem leads to a self-similarly evolving peaked void size distribution.
\end{abstract}

\section{Introduction: Excursions}

Hierarchical scenarios of structure formation have been very succesfull in understanding the formation histories of gravitationally bound virialized haloes. Particularly compelling has been the formulation of a formalism in which the collapse and virialization of overdense dark matter halos within the context of hierarchical clustering can be treated on a fully analytical basis. This approach was originally proposed by Press \& Schechter (1974), which found a particularly useful and versatile formulation and modification in the the excursion set formalism (Bond et al. 1991).

It is based on the assumption that for a structure to reach a particular nonlinear evolutionary stage, such as complete gravitational collapse, the sole condition is that its linearly extrapolated primordial density should attain a certain value. The canonic example is that of a spherical tophat overdensity collapsing once it reaches the collapse barrier $\delta_{c} \approx 1.69$. The successive contributions to the local density by perturbations on a (mass) resolution scale $S_{m}$ may be represented in terms of a density perturbation random walk, the cumulative of all density fluctuations at a resolution scale smaller than $S_{m}$. By identifying the largest scale at which the density passes through the barrier $\delta_{c}$ it is possible (1) to infer at any cosmic epoch the mass spectrum of collapsed halos and (2) to reconstruct the merging history of each halo (see Fig. 2] top right).

In this study we demonstrate that also the formation and evolution of foamlike patterns as a result of the gravitational growth of primordial density pertur- 

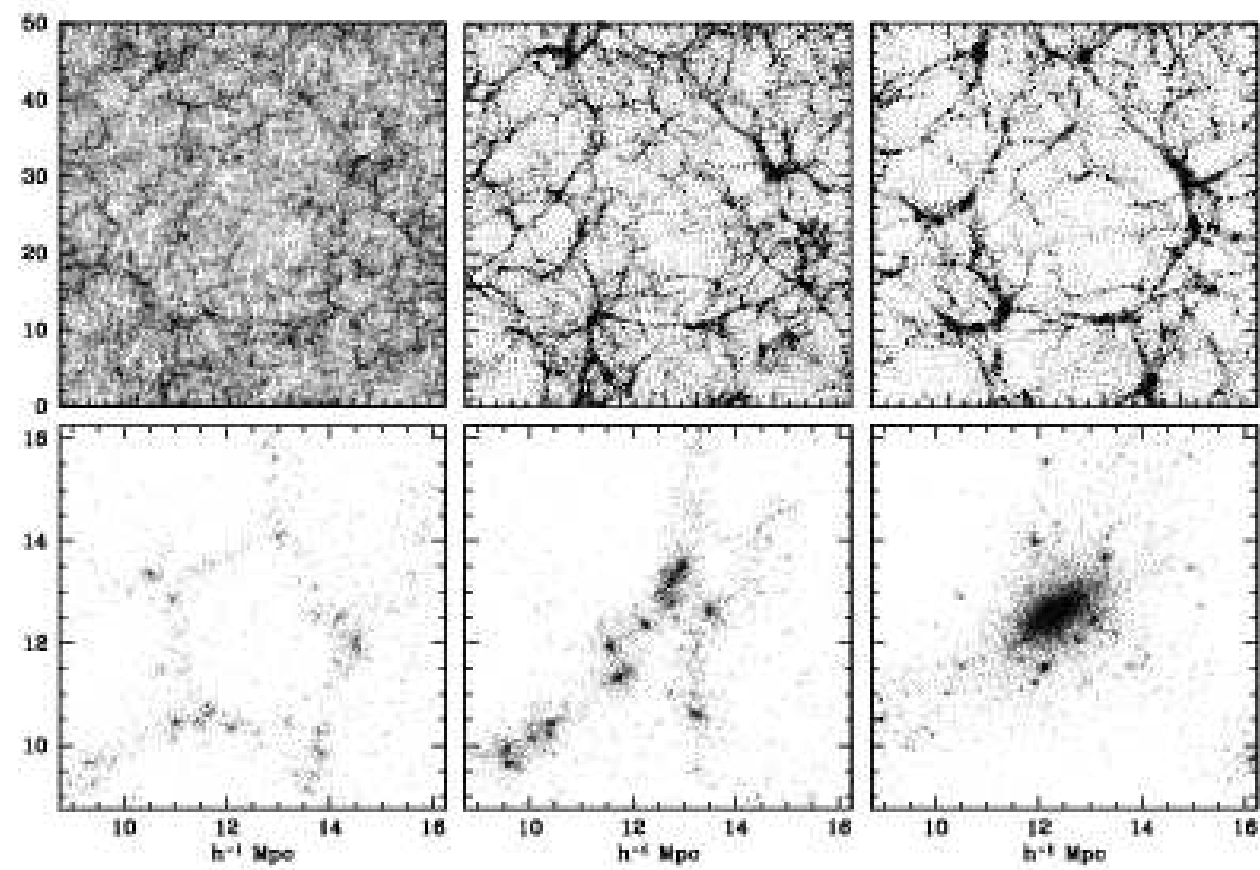

Figure 1. Illustration of the two essential "void hierarchy modes": (top) the void-in-void process (top), with a void growing through the merging of two or more subvoids; (bottom) the void-in-cloud process: a void demolished through the gravitational collapse of embedding region.

bations is liable to a succesfull description by the excursion set analysis. This is accomplished by resorting to a complementary view of clustering evolution in which we focus on the evolution of the voids in the Megaparsec galaxy and matter distribution, spatially the dominant component. An extensive description of this work can be found in Sheth \& Van de Weygaert (2003, MNRAS, submitted).

\section{Void Evolution: the two processes}

Primordial underdensities are the progenitors of voids. Because underdensities are regions of suppressed gravitational attraction, representing an effective repulsive gravity, matter flows out of their interior and moves outward to the edges of these expanding voids. Voids expand, become increasingly empty and develop an increasingly spherical shape (Icke 1984). Matter from the void's interior piles up near the edge: usually a ridge forms around the void's rim and at a characteristic moment the void's interior shells take over the outer ones. At this shell-crossing epoch the void reaches maturity and becomes a nonlinear object expanding self-similarly, the implication being that the majority of observed voids is at or near this stage (Blumenthal et al. 1992). As voids develop from underdensities in the primordial cosmic density field, the interaction with internal substructure and external surrounding structures translates into a con- 
tinuing process of hierarchical void evolution (Dubinski et al. 1993, Van de Weygaert \& Van Kampen 1993).

The evolution of voids resembles that of dark halos in that large voids form from mergers of smaller voids that have matured at an earlier cosmic time (Fig. 1 top row). However, in contrast to dark halos, the fate of voids is ruled by two processes. Crucial is the realization that the evolving void hierarchy does not only involve the void-in-void process but also an additional aspect, the void-in-cloud process. Small voids may not only merge into larger encompassing underdensities, they may also disappear through collapse when embedded within a larger scale overdensity (Fig. 11 bottom row). In terms of the excursion set approach, it means that the one-barrier problem for the halo population has to be extended to a more complex two-barrier problem. Voids not only should ascertain themselves of having decreased their density below the void barrier $\delta_{v}$ of the shell-crossing transition. For their survival and sheer existence it is crucial that they take into account whether they are not situated within a collapsing overdensity on a larger scale which crossed the collapse barrier $\delta_{c}$. They should follow a random walk path like type "3" in Fig.2 (bottom right), rather than the void-in-cloud path " 4 ". The repercussions of this are far-reaching and it leads to a major modification of the void properties and distribution.
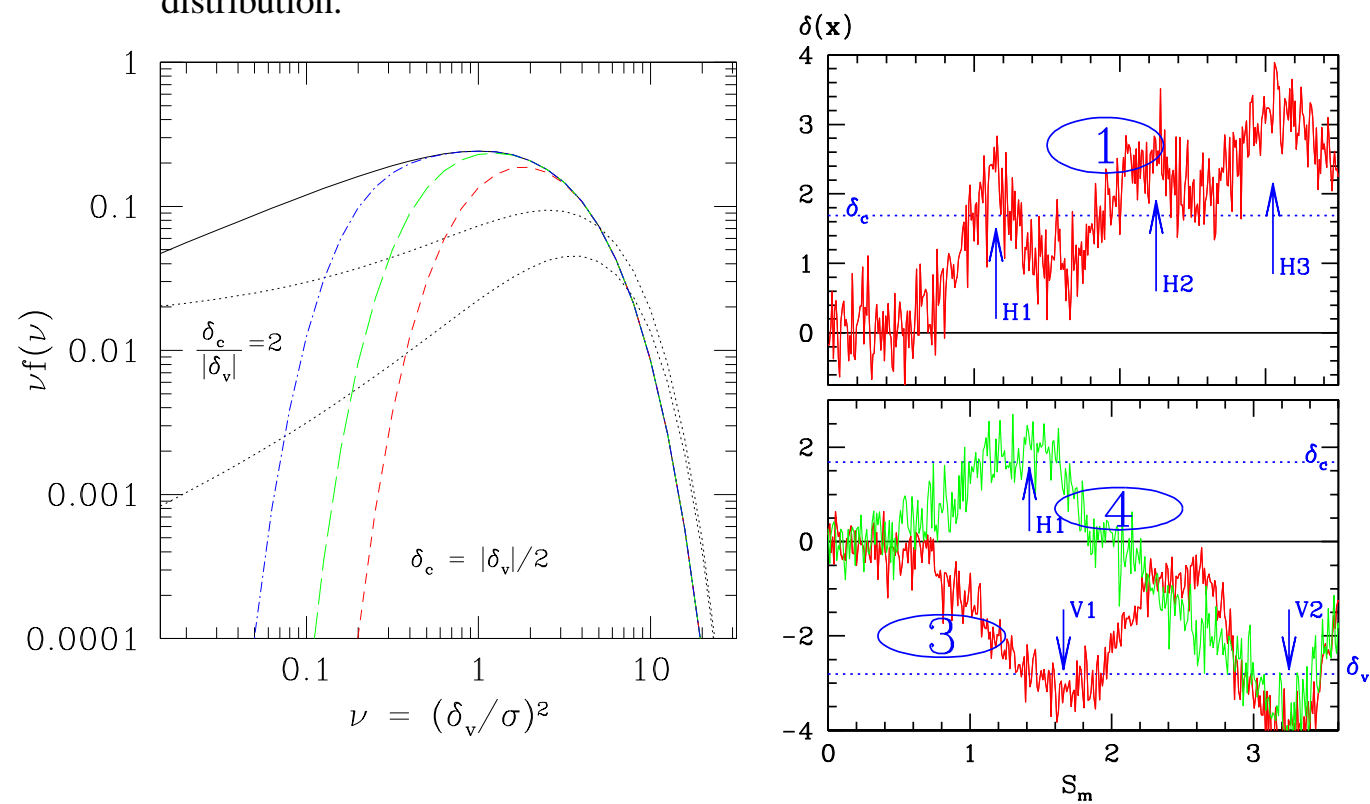

Figure 2. Left: Void Size Probability Function for various values of the void-and-cloud parameter $\delta_{c} / \delta_{v}: 1 / 2$ (short dashed), 1 (dashed), 2 (dot-dashed), and $\infty$ (solid). For reference, the dotted curves are predictions by the "adaptive peaks model" of Appel \& Jones (1990). Right: Excursion Set Random Walks, local density $\delta(\mathbf{x})$ as function of mass resolution $S_{m}$. Clearly, the fluctuations are larger as resolution $S_{m}$ increases. (Top) Halo formation. Horizontal dashed bar is the collapse barrier $\delta_{c}$. (Bottom) Fate of void V2, illustrated by 2 possible random walks: curve " 4 " is a void-in-cloud process, curve " 3 " a void-in-void process. 


\section{Void Distribution: Universal, Peaked and Self-Similar}

Analytically, the resulting expression follows by evaluating the fraction of walks which first cross $\delta_{\mathrm{v}}$ at $S$, and which do not cross $\delta_{\mathrm{c}}$ until after they have crossed $\delta_{\mathrm{v}}$ (Sheth \& Van de Weygaert 2003). An insightful approximation of this distribution, in terms of the "self-similar" void density $\nu \equiv \delta_{\mathrm{v}}^{2} / S$ is

$$
\nu f(\nu) \approx \sqrt{\frac{\nu}{2 \pi}} \exp \left(-\frac{\nu}{2}\right) \exp \left(-\frac{\left|\delta_{\mathrm{v}}\right|}{\delta_{\mathrm{c}}} \frac{D^{2}}{4 \nu}-2 \frac{D^{4}}{\nu^{2}}\right),
$$

in which the $D \equiv\left(\left|\delta_{\mathrm{v}}\right| /\left(\delta_{\mathrm{c}}-\delta_{\mathrm{v}}\right)\right.$ parameterizes the the relative impact of void and halo evolution on the hierarchically evolving population of voids.

The resulting distributions, for various values of $D$, are shown in Figure 2 (left). The void size distribution is clearly peaked about a characteristic size: the void-in-cloud mechanism is responsible for the demise of a sizeable population of small voids. The halo mass distribution diverges towards small scale masses, so that in terms of numbers the halo population is dominated by small mass objects. The void population, on the other hand, is "void" of small voids and has a sharp small-scale cut-off.

Four additional major observations readily follow from this analysis: $(\bullet)$ At any one cosmic epoch we may identify a characteristic void size which also explains why in the present-day foamlike spatial galaxy distribution voids of $\sim 20-30 h^{-1} \mathrm{Mpc}$ are the predominant feature; $(\bullet)$ The void distribution evolves self-similarly and the characteristic void size increases with time: the larger voids present at late times formed from mergers of smaller voids which constituted the dominating features at earlier epochs (Fig. 11 top panels); (•) Volume integration shows that at any given time the population of voids approximately fill space, apparently squeezing the migrating high-density matter in between them; (•) As the size of the major share of voids will be in the order of that of the characteristic void size this observation implies that the cosmic matter distribution resembles a foamlike packing of spherical voids of approximately similar size and excess expansion rate.

In all, a slight extension and elaboration on the original extension formulism enables the framing of an analytical theory explaining how the characteristic observed weblike Megaparsec scale galaxy distribution, and the equivalent frothy spatial matter distribution seen to form in computer simulations of cosmic structure formation, are natural products of a hierarchical process of gravitational clustering. A continuously evolving hierarchy of voids produces a dynamical foamlike pattern whose characteristic dimension grows continuously along with the evolution of cosmic structure, a Universe which at any one cosmic epoch is filled with bubbles whose size corresponds to the scale just reaching maturity. 